\title{
LOGROS Y RETOS DE LA IMPLEMENTACIÓN DE DERIVADOS FINANCIEROS ENTANDARIZADOS EN COLOMBIA: UN ANÁLISIS COMPARATIVO CON MÉXICO, CHILE Y PERÚ, 2008-2012
}

\author{
DIANA M. CARMONA MUÑOZ2; DANNA J. HIGUERA HURTADO; ANA P. ESPELETA BAYONA ${ }^{4}$ \\ UNIVERSIDAD DE LA SALLE
}

Recibido, agosto 11 de 2014

Concepto evaluación, agosto 20 de 2014

Aceptado, septiembre 30 de 2014

\begin{abstract}
Referencia: Gutiérrez Mendoza, L.; Ariza Nieves (2014). "Logros y retos de la implementación de derivados financieros estandarizados en Colombia: un análisis comparativo con México, Chile y Perú, 20082012”. Revista Academia y Virtualidad, 7, (2), 76 -87
\end{abstract}

\section{Resumen}

Desde 1999 con la eliminación del sistema de banda cambiaria inicia un desarrollo significativo en el mercado de derivados colombiano (отс) $)^{5}$. Con las recientes crisis financieras internacionales, aumenta la necesidad de estandarizar los productos derivados financieros con el fin de eliminar el riesgo de contraparte presentado en el mercado отC. Este artículo identifica los logros y los retos que presenta el mercado de derivados estandarizado en Colombia desde su creación en 2008 hasta 2012, analizando así años completos; se compara su evolución con la que ha venido presentado México, Chile y Perú en el mismo periodo mediante una matriz de diagnóstico para posteriormente plantear estrategias de desarrollo de mercado, desarrollo de producto, integración horizontal, acceso al mercado y desarrollo de negocio por medio de la adaptación del plan estratégico mexicano al mercado colombiano.

Palabras clave: derivados, integración horizontal, mercado estandarizado, riesgo financiero.

\section{ACHIEVEMENTS AND CHALLENGES OF THE IMPLEMENTATION OF DERIVATIVES FINANCIAL STANDARDIZED IN COLOMBIA: A COMPARATIVE ANALYSIS WITH MEXICO, CHILE AND PERU, 2008-2012}

\begin{abstract}
Since 1999 with the elimination of exchange rate band system initiates a significant development in the OTC derivatives market in Colombia. With the recent international financial crisis increases the need for standardized financial de-

1. Este artículo es resultado de la investigación "Logros y retos de la implementación de derivados financieros estandarizados en Colombia frente a los demás países pertenecientes al MILA: un análisis comparativo”, Grupo de Investigación en Mercados y Desarrollo Empresarial Global.

2. Magíster, Finanzas, Universidad Externado de Colombia; Especialista, Finanzas y Mercado de Capitales, Universidad de La Sabana; Economista, Universidad de La Salle; docente-investigadora, Universidad de La Salle.

3. Profesional, Finanzas y Comercio Internacional, Universidad de la Salle.

4. Profesional, Finanzas y Comercio Internacional, Universidad de la Salle.

5. Over the Counter (OTC): Mercado de instrumentos financieros negociados fuera del ámbito de las bolsas de valores formales; también conocido como mercado extra bursátil o no estandarizado.
\end{abstract}


Logros y retos de la implementación de derivados financieros entandarizados en colombia: un análisis comparativo con méxico, chile y perú, 2008-2012

rivatives in order to eliminate counterparty risk in the OTC market presented. This article identifies the achievements and challenges of standardized derivatives market in Colombia since its inception in 2008 until 2012 and analyzing full years; evolution compared to that presented in Mexico, Chile and Peru by an array of diagnostic subsequently raise strategies: market development, product development, horizontal integration, market access and business development through adaptation plan Mexican strategic to the Colombian market.

Keywords: derivatives, horizontal integration, market standardized, financial risk.

\section{LOGROS E RETOS DA IMPLEMENTAÇÃO DE DERIVADOS FINANCEIROS ENTANDARDIZADOS NA COLÔMBIA: UMA ANÁLISE COMPARATIVA COM O MÉXICO, O CHILE E O PERU, 2008-2012}

\section{Resumo}

Desde 1999 com a eliminação do sistema de banda cambiária dá-se início a um desenvolvimento significativo no mercado de derivados colombiano. (OTC)4 Com as recentes crises financeiras internacionais, cresce a necessidade de estandardizar os produtos derivados financeiros com fim de eliminar o risco de contraparte apresentado no mercado OTC. Neste artigo identificam-se os logros e os retos que apresenta o mercado de derivados estandardizado na Colômbia desde sua criação em 2008 até 2012, analisando assim anos completos; compara-se seu desenvolvimento com o que têm vindo apresentando o México, o Chile e o Peru no mesmo período por meio de uma matriz de diagnóstico para seguidamente plantear estratégias de desenvolvimento de mercado, desenvolvimento de produto, integração horizontal, acesso ao mercado e desenvolvimento de negócio por meio da adaptação do plano estratégico mexicano ao mercado colombiano.

Palavras-chave: derivados, integração horizontal, mercado estandardizado, risco financeiro.

\section{Introducción}

El mercado de derivados en Colombia según cifras oficiales del Banco de la República de Colombia (20032012) ha incrementado de manera significativa el número de operaciones; con la expedición de la Ley 964 de 2005, se genera una consolidación del marco regulatorio institucional con respecto al mercado de valores, ofreciendo mayor seguridad jurídica, claridad y mejores prácticas de gobierno corporativo. Según Córdoba (2008), presidente de la Bolsa de Valores de Colombia ${ }^{6}$, "la creación de este mercado de derivados estandarizados es un avance significativo para el país, pues ofrece la oportunidad a la gente de invertir en un derivado de un instrumento financiero, con la posibilidad de administrar el riesgo al que están expuestos los activos”.

Con la presente investigación se busca evaluar la evolución del mercado de derivados financiero estandarizado en cada uno de los países objeto de estudio, centrando la atención en Colombia, e identificando así las ventajas y desventajas que el país presenta frente a los demás países, con el fin de formular recomendaciones en cuanto a la implementación o modificación de la estructura operacional y regulatoria del mercado de derivados, de tal manera que se ajuste a las prácticas internacionales, permitiendo un mayor desarrollo y aumento en las operaciones de derivados, para así poder plantear estrategias y medidas que se deben

6. En adelante BVC. 
Diana M. Carmona Muñoz ; Danna J. Higuera Hurtado ; Ana P. Espeleta Bayona

tener en cuenta para mejorar el mercado de derivados en Colombia.

\section{Antecedentes y referentes teóricos}

Teniendo en cuenta la reciente incorporación al mercado de derivados de los países contemplados en este estudio, así como la limitada información publicada respecto a este tema, se hace necesario analizar estudios, investigaciones e informes que, aunque no son recientes, contribuyen al desarrollo de este proceso investigativo. Por esto, se toman en cuenta estudios de hace más de 10 años.

Por una parte, se destacan estudios importantes como el realizado por Salazar (2008, p. 28), vicepresidente de negocios internacionales del Banco de Bogotá, quien en su investigación acerca del mercado de derivados busca mostrar la evolución de las negociaciones de derivados en el mercado global OTC, mediante una descripción cualitativa y cuantitativa. Éste afirma que, desde el punto de vista de los agentes del sector real $^{7}$, el conocimiento y difusión de los derivados en Colombia es aún limitado, por lo cual se requiere un esfuerzo mancomunado de cada uno de los actores interesados para entender mejor estos instrumentos. Asimismo, Salazar concluye que uno de los principales impedimentos para el desarrollo de los mercados de derivados es el factor legal e institucional que genera gran heterogeneidad en los mercados de la región; adicionalmente afirma que las limitantes con las que cuentan las empresas colombianas para ingresar al mercado de derivados, se reflejará en una menor competitividad en el mercado global.

Un estudio posterior al de Salazar, relevante en el tema de investigación es el realizado por $\operatorname{DECEVAL}^{8}$ y ANIF $^{9}$ (2009, p.3), quienes -mediante un análisis comparativoencontraron que en Colombia el mercado de derivados se encuentra en una fase de desarrollo similar a la que vivieron México y Brasil hace una década, donde la estabilidad macroeconómica, la reducción de la inflación y la consolidación de políticas fiscales ortodoxas, constituyen instrumentos fundamentales en el desarrollo de los mercados. Deceval y ANIF también encontraron que la Ley 964 de 2005 (Congreso de Colombia, 2007) marcó un precedente importante en el esquema del mercado de derivados, particularmente el decreto 1796 de 2008 (Superfinanciera, 2008), el cual sentó las bases de la implementación del sistema de derivados en Colombia; adicionalmente, muestran cómo se ha venido desarrollando un importante mercado de derivados estandarizados de energía eléctrica, debido a que el sector eléctrico colombiano cuenta con contratos bilaterales no estandarizados que generan altos riesgos de contraparte, generando así la necesidad de realizar contratos de cobertura.

Ahora bien, respecto a las investigaciones realizadas para el mercado de derivados financieros en Chile sobresale el estudio realizado por Ochoa \& Benjerodt (1996, p. 43); miembros del departamento de investigaciones económicas del Banco Central de Chile, quienes mediante un análisis cualitativo y cuantitativo de los elementos legales-institucionales de la normatividad cambiaria del país, así como de las regulaciones tributarias y de las perspectivas del mercado doméstico, concluyen que para el proceso de liberalización y globalización financiera se visualizaba una rápida expansión del mercado interno de derivados financieros para los próximos años, instrumentos que eventualmente se trasformarían en una de las líneas de negocio y de toma de riesgo más importante de las instituciones financieras residentes y de otros inversionistas; de igual manera, esta investigación determinó que se generalizaría el conocimiento de las ventajas de los instrumentos para la protección de los riesgos de mercado a los que estarían expuestos sus portafolios, en un ámbito de acrecentada volatilidad de

7. Sector real: empresas industriales y comerciales que necesitan recursos financieros para adelantar su tarea productiva.

8. DECEVAL: Depósito Centralizado de Valores de Colombia. Entidad facultada para recibir en depósito títulos valores, instrumentos financieros y valores que se encuentren o no inscritos en el registro nacional de valores e intermediarios.

9. ANIF: Asociación Nacional de instituciones financieras. Gremio de opinión encargado de realizar estudios, divulgar documentos y difundir ideas y propuestas por medio de sus seminarios. 
precios de activos y pasivos. Este estudio ha acertado en la manera como los derivados se han expandido considerablemente en el mercado chileno. En la actualidad se negocian instrumentos derivados financieros con el fin de generar confianza, los cuales buscan minimizar o neutralizar completamente el efecto del movimiento de los precios de los activos y los riesgos que existen en el mercado.

La investigación realizada por Budnevich y Asociados ${ }^{10}$ (2009, p. 84) compara el mercado de derivados de Chile con los principales mercados de derivados del mundo (EE.UU, Inglaterra, México, Brasil, Hong Kong y la experiencia en países asiáticos), identificando las mayores deficiencias del mercado de derivados en Chile, y estructurando propuestas para contribuir al desarrollo del mercado, enfatizándose en el mercado de bolsas versus отC, un mejor manejo de riesgo de contraparte y la neutralidad tributaria y de instrumentos.

A diferencia de Colombia y Chile, Perú ha sido un mercado menos desarrollado en cuanto a la implementación de productos derivados financieros, esto se refleja en los pocos estudios económicos que se han realizado respecto al tema. Una de las investigaciones es desarrollada por Bernedo \& Azareño (2003, p.27), quienes exponen los elementos y condiciones necesarias para el desarrollo de un mercado de derivados, y por medio de un análisis descriptivo identifican los elementos necesarios para una adecuada arquitectura del mercado, debido al grado primitivo en que se encuentra el mercado de derivados en el Perú; llegando a concluir que la implementación de estos instrumentos financieros contribuirá a la acumulación de reservas internacionales y estabilización del tipo de cambio; adicionalmente, muestran la efectividad que México y Colombia han tenido en la emisión de opciones, tanto en el incremento de sus reservas como en la mitigación de los efectos negativos de las fuertes depreciaciones.
De acuerdo con Saavedra \& Utrilla (2010, p. 309), el crecimiento de los instrumentos de derivados en México ha sido muy significativo; sin embargo, se aprecia que la mayor parte de operaciones realizadas por los bancos obedece a actividades de especulación, por ello se hace necesario reforzar las medidas de control y trasparencia en su utilización considerando la alta vulnerabilidad que se tienen frente a ataques especulativos.

De otro lado, la teoría de derivados, a diferencia de otros temas financieros, es relativamente nueva, a pesar de que el uso de estos instrumentos data de la Edad Media (476 a 1492), cuando fueron creados los primeros futuros que originalmente satisfacían las demandas de agricultores y comerciantes, con el fin de eliminar para ambas partes el riesgo que suponía el precio futuro incierto de los productos agrícolas (Hull; 2002, p.2).

El Banco de la República de Colombia define los derivados como contratos financieros cuyo valor depende del precio de un activo, de una materia prima o de la evolución de una tasa de interés, en donde el activo, la materia prima o tasa de interés de que depende o se deriva el contrato toma el nombre de subyacente. Los instrumentos derivados tradicionales son los forwards, futuros, opciones y swaps, los cuales permiten transferir el riesgo a quienes estarían dispuestos o en mejor capacidad para asumirlos. Además de los derivados tradicionales existen los derivados de crédito, que también cumplen la función de transferir el riesgo, en este caso el riesgo crediticio (Gomez, 2010).

Los productos derivados constituyen unos de los grandes éxitos de la economía financiera moderna; algunas de sus principales funciones son asegurar precios futuros en aquellos mercados volátiles, neutralizar los riesgos de fluctuación en las tasas de interés, reducir costos de transacciones y de reasignación de activos, crear vías agiles para el arbitraje entre mercados permitiendo alinear precios de los instrumentos de deuda, acciones

10. Investigación elaborada por Budnevich y Asociados Limitada para la Superintendencia de Bancos e Instituciones Financieras y la Superintendencia de Valores y Seguros. Los economistas Carlos Budnevich y Salvador Zurita elaboraron este informe. 
Diana M. Carmona Muñoz ; Danna J. Higuera Hurtado ; Ana P. Espeleta Bayona

y derivados, incrementando así la eficiencia y liquidez en sus respectivos mercados; adicionalmente, permite obtener o prestar recursos financieros a tasas más favorables, realizar con mayor eficiencia operaciones de venta en corto de valores y controlar costos asociados con márgenes (Díaz \& Hernández, 2003).

\section{Diagnóstico: ventajas y desventajas de Colombia frente a los demás países}

A continuación se muestra un diagnóstico, a través del cual se identifican las ventajas y las desventajas de cada uno de los mercados, con el fin de mirar la posición que tiene Colombia frente a estos países y, posteriormente, plantear estrategias encaminadas a profundizar el mercado de derivados estandarizado en Colombia.

\subsection{Volúmenes de negociación}

\subsubsection{Colombia}

Volúmenes de negociación ( $\mathbf{N}^{\circ}$ de contratos anuales): mínimo 2008: 621, máximo 2011: 795.096

Ventajas: Al ser un mercado nuevo con crecimientos importantes genera atracción a clientes potenciales.

Desventajas: Bajos volúmenes de negociación respecto al mercado de derivados de México.

\subsubsection{Chile}

Volúmenes de negociación ( $\mathrm{N}^{\circ}$ de contratos anuales): no se encuentran estadísticas en fuentes oficiales.

No se pueden identificar las ventajas y desventajas en cuanto a volúmenes de negociación, ya que no se dispone de la información para el respectivo análisis.

\subsubsection{Perú}

Volúmenes de negociación ( $\mathrm{N}^{\circ}$ de contratos anuales): no ha desarrollado mercado estandarizado

Ventajas: no está expuesto a riesgo de liquidez, causado por la negociación de productos derivados.

Desventajas: cierra oportunidades a fuentes potenciales de ingresos para el mercado estandarizado

\subsubsection{México}

Volúmenes de negociación ( $\mathrm{N}^{\circ}$ de contratos anuales): mínimo 2010: 42.599.595, máximo 2008: 70.147.706.

Ventajas: sus significativos niveles de negociación generan alta liquidez al mercado y atraen mayor inversión. Desventajas: se expone a altos niveles de riesgo, en especial si los instrumentos de derivados financieros se utilizan de manera inadecuada

\subsection{Tipos de contratos negociados}

\subsubsection{Colombia}

Futuros sobre: acciones, renta fija, divisas, tasas de interés e índices bursátiles.

Ventajas: tiene diversidad de contratos a futuro, siendo éste un mercado tan líquido; generalmente, siempre existe un comprador o vendedor dispuesto aceptar una orden del mercado.

Desventajas: el mercado estandarizado en Colombia se limita al manejo de futuros; por tanto, no ofrece un portafolio diversificado en otros tipos de contratos como opciones y swaps.

\subsubsection{Chile}

Futuro sobre índice bursátil IPSA.

Ventajas: este futuro toma como activo subyacente el índice de precio selectivo de Chile, el cual mide el resultado de las compañías más líquidas del mercado.

Desventajas: al presentar sólo contratos a futuro sobre el IPSA, no genera un portafolio diversificado para los inversionistas, sino - por el contrario- desvía su atención a otros mercados.

\subsubsection{Perú}

No ha desarrollado mercado estandarizado

Ventajas: puede fortalecer el mercado отC, el cual se ajusta a las necesidades de las partes; no necesita un mercado establecido y permite la negociación de los precios.

Desventajas: al no desarrollar un mercado estandarizado y centrarse en el OTC, genera mayores riesgos de incumplimiento del contrato pactado. 
Logros y retos de la implementación de derivados financieros entandarizados en colombia: un análisis comparativo con méxico, chile y perú, 2008-2012

\subsubsection{México}

Futuros sobre: acciones, renta fija, divisas, tasas de interés e índices bursátiles. Opciones sobre: divisas, índices y acciones. Swaps sobre: renta fija

Ventajas: ofrece un amplio número de instrumentos derivados, generando mayor liquidez y profundidad del mercado, además de la atracción de inversionistas que buscan un portafolio diversificado tanto en tipos de contrato como en activo subyacente.

Desventajas: el mercado requiere mayor control y supervisión, al contar con diversidad de contratos, además de exponerse a altos niveles de capital golondrina, ya que el portafolio diversificado atrae en gran medida a especuladores.

\subsection{Miembros del mercado de derivados estandarizado (a julio de 2013)}

\subsubsection{Colombia}

Miembros liquidadores y no liquidadores: 39

Ventajas: comparado con México, Colombia tiene un número importante de miembros teniendo en cuenta que los volúmenes de su mercado son muchos menores a los que presenta México, esto genera para Colombia un desarrollo más eficiente de su mercado.

Desventajas: pese a que el aumento en el número de miembros del mercado genera mayor liquidez y profundidad de éste, las entidades reguladoras tendrán una labor más compleja de control y supervisión por lo que deberán realizar su tarea de vigilancia de manera cuidadosa. Este escenario puede incrementar los costos del mercado.

\subsubsection{Chile}

Las fuentes oficiales no registran la cantidad de miembros que permiten el desarrollo y fortalecimiento del mercado a futuro del IPSA.

\subsubsection{Perú}

No ha desarrollado mercado estandarizado

Ventajas: teniendo en cuenta el contexto internacional y la tendencia a invertir en mercados emergentes, Perú podría aprovechar este escenario para dar sus primeros pasos en el desarrollo del mercado de derivados estandarizado iniciando con el fortalecimiento y adecuación de los miembros del mercado.

Desventajas: los miembros pertenecientes al mercado de valores en el Perú centran sus operaciones en el mercado de renta variable y renta fija, y al no tener un mercado de derivados estandarizado los miembros limitan sus operaciones y pierden ventajas competitivas frente a los miembros de los demás países.

\subsubsection{México}

Socios liquidadores: 5 , operadores: 53 , formadores de mercado: 17 , miembros totales: 75

Ventajas: de los mercados analizados es el que tiene más miembros para su operación, lo cual se ve reflejado en sus altos volúmenes de negociación. Su importante número de formadores de mercado favorece las condiciones de liquidez y crecimiento del mercado.

Desventajas: se presenta mayor dificultad de supervisión y vigilancia del mercado en lo que respecta a esquemas operativos, funciones y obligaciones de los participantes del mercado.

\subsection{Marco normativo}

\subsubsection{Colombia}

Marco normativo

Ventajas: las frecuentes actualizaciones normativas y la adaptación que realiza el mercado, han permitido la inclusión de diferentes tipos de futuros; se espera que este trato normativo se realice para opciones y swaps.

Desventajas: marco normativo centrado en la negociación de futuros, limitando la inclusión de opciones y swaps en el mercado.

\subsubsection{Chile}

Marco normativo

Ventajas: a pesar de que sólo posee una clase de contrato a futuro sobre un único subyacente, el mercado presenta un marco regulatorio bien definido en lo que respecta a negociaciones IPSA.

Desventajas: al centrar su marco normativo en la 
negociación de futuros IPSA, limita la entrada tanto de otros contratos a futuro, como de instrumentos de opciones y swaps.

\subsubsection{Perú}

Marco normativo

Ventajas: un marco normativo que no presenta ninguna ventaja en el desarrollo del mercado de derivados estandarizado.

Desventajas: sólo cuenta con una ley que regula el mercado de derivados financieros, la cual es bastante básica y no incluye operaciones en el mercado estandarizado.

\subsubsection{México}

Marco normativo completamente estructurado

Ventajas: de los países analizados es el que tiene el marco normativo más estructurado, generando así mayor protección tanto para el mercado como para los inversionistas.

Desventajas: se puede generar dificultad en el conocimiento de la normatividad que aplica en el mercado, en especial en lo que respecta a pequeños inversionistas.

\subsection{Restricción a la inversión extranjera}

\subsubsection{Colombia}

Impuestos sobre ganancias: $14 \%$.

Ventajas: el proceso de reducción en la carga impositiva que ha venido realizando Colombia, atrae mayor inversión e ingresos, generando la posibilidad de invertir estos recursos en el desarrollo y fortalecimiento del mercado.

Desventajas: al tener el mayor impuesto a la inversión extranjera con respecto a los demás países analizados, el mercado pierde competitividad; pese al proceso de reducción impositiva que ha venido presentando, aún no es suficiente para competir de forma equitativa con los demás mercados.

\subsubsection{Chile}

Impuestos sobre ganancias: exentos.

Ventajas: exime del pago de impuesto a los inversionistas extranjeros, generándoles mayores atractivos para invertir en el mercado.

Desventajas: al no tener un mercado estandarizado o estructurado que contemple un portafolio diversificado para el inversionista, no puede aprovechar la ventaja competitiva con la que cuenta en el aspecto tributario.

\subsubsection{Perú}

Impuestos sobre ganancias: 5\%

Ventajas: posee una ventaja competitiva con respecto a Colombia, ya que la carga impositiva a la inversión extranjera es menor.

Desventajas: pese a que el mercado peruano se encuentra en una etapa de exploración, cuenta con una desventaja competitiva frente a Chile y México, ya que estos dos países eximen del cobro de impuesto a los inversionistas.

\subsubsection{México}

Impuestos sobre ganancias: exentos

Ventajas: además de ser uno de los mercados más completos de la región, la exención tributaria a los inversionistas extranjeros hace mucho más llamativo este mercado.

Desventajas: la entrada de inversión extranjera en exceso al país, puede provocar procesos de revaluación de la moneda local, e incrementos en capitales golondrina ${ }^{11}$.

\section{Matriz de la gran estrategia para el merca- do de derivados estandarizado en Colombia.}

La matriz es la Gran Estrategia, cuyo objetivo consiste en generar recomendaciones a partir del diagnóstico realizado previamente; de acuerdo con la Universidad Nacional de Manizales (2012), esta matriz se basa en dos dimensiones evaluativas: posición competitiva y crecimiento del mercado; los diferentes aspectos de las variables analizadas, podrán ubicarse en cada uno de los cuadrantes dependiendo del grado de competitividad $\mathrm{y}$ de crecimiento que presenten dentro del mercado de derivados financiero colombiano.

11. Capital que llega al país, permanece por un poco tiempo y luego sale del país nuevamente. 
Las variables ubicadas en el cuadrante I de la matriz cuentan con una excelente posición estratégica; de acuerdo con Fred (2003, p.215), estas variables pueden aprovechar satisfactoriamente las oportunidades externas, pueden correr riesgos agresivos en caso de requerirlo y se consolidan como factores claves para el desarrollo del mercado, por lo cual las estrategias propuestas en este cuadrante están enfocadas a continuar aprovechando las ventaja competitivas ya establecidas.

de estar en un contexto de mercado creciente, de tal manera que las estrategias establecidas en este cuadrante se enfocan en mejorar la competitividad del mercado, teniendo en cuenta las grandes oportunidades que presenta el importante desarrollo del mercado.
Las variables ubicadas en el cuadrante III generan un crecimiento lento del mercado de derivados, además de una posición competitiva débil, lo cual implica que éstos son los factores más críticos dentro del desarrollo del mercado, de tal manera que se establecen estrategias radicales que cambien el rumbo del mercado con el objetivo final de llegar al cuadrante I, generando una posición competitiva fuerte $\mathrm{y}$ un rápido crecimiento del mercado.

Finalmente, las variables ubicadas en el cuadrante IV a pesar de que han generado una posición competitiva fuerte no han permitido el crecimiento rápido del mercado; en consecuencia, las estrategias recomendadas en este cuadrante están enfocadas al aprovechamiento de

\begin{tabular}{|c|c|c|c|}
\hline \multirow{6}{*}{ 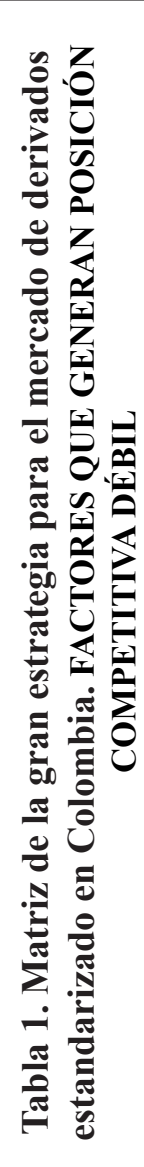 } & \multicolumn{2}{|c|}{ FACTORES QUE GENERAN CRECIMIENTO RÁPIDO DEL MERCADO } & \multirow{4}{*}{ 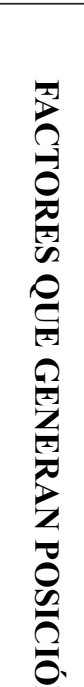 } \\
\hline & CUADRANTE II & CUADRANTE I & \\
\hline & $\begin{array}{l}\text { 1. Desarrollo del mercado: normatividad e } \\
\text { incremento de los miembros. } \\
\text { 2. Desarrollo del producto: diversificación } \\
\text { de los tipos de contratos negociados } \\
\text { 3. Integración Horizontal: búsqueda de } \\
\text { integración con los principales mercado } \\
\text { de derivados estandarizados de la región. }\end{array}$ & $\begin{array}{l}\text { 1. Penetración del mercado: volúmenes de } \\
\text { negociación. Crecimientos anuales superiores al } \\
1000 \%\end{array}$ & \\
\hline & CUADRANTE III & CUADRANTE IV & \\
\hline & $\begin{array}{l}\text { 1. Acceso al mercado de derivados: } \\
\text { mejorar la competitividad en cuanto a } \\
\text { tributación de inversionistas extranjeros. } \\
\text { 2. Estrategia a nivel de negocios: creación } \\
\text { de un plan estratégico donde Colombia } \\
\text { defina los objetivos a corto, mediano } \\
\text { y largo plazos, asignados los recursos } \\
\text { requeridos }\end{array}$ & $\begin{array}{l}\text { Los factores en los que Colombia posee una } \\
\text { posición competitiva fuerte, han generado un } \\
\text { rápido crecimiento del mercado, ubicándose en } \\
\text { el cuadrante I. }\end{array}$ & 20 \\
\hline & \multicolumn{2}{|c|}{ FACTORES QUE GENERAN CRECIMIENTO LENTO DEL MERCADO } & \\
\hline
\end{tabular}


la posición competitiva en pro de un mayor crecimiento del mercado.

La matriz de la Gran Estrategia, por consiguiente, se ha convertido en uno de los instrumentos más usados al momento de formular estrategias alternativas.

\section{Estrategias}

La mayoría de variables se ubican en el cuadrante II, debido a que el mercado de derivados estandarizado colombiano se encuentra en un proceso de crecimiento rápido, pero posee factores que limitan su competitividad. En el cuadrante I se ubican los logros que ha tenido el mercado, mientras que en los cuadrantes II, III y IV se encuentras los retos del mercados, para los cuales se han establecido estrategias y recomendaciones que debería adoptar el mercado de derivados estandarizado en Colombia.

\subsection{Cuadrante I}

\subsubsection{Penetración del mercado}

En esta instancia, se plantea una estrategia de penetración de mercado enfocada en el conocimiento y educación de los derivados financieros estandarizados, especialmente en las pequeñas y medianas empresas colombianas que realizan operaciones de importación y/o exportación, o aquellas que tienen deuda en moneda extranjera, debido a que éstas constituyen la principal fuente de generación de empleo, son parte fundamental del sistema económico, estimulan la economía, generan más del $50 \%$ del empleo nacional y representan el $40 \%$ del producto interno bruto del país (Jiménez , 2012).

El conocimiento del mercado de derivados estandarizado permitirá a los empresarios entender los beneficios que en términos de cobertura genera el mercado, ya que reduce la incertidumbre de los resultados de la empresa y elimina el riesgo de contraparte por medio de la cámara de compensación, escenario que no se presenta en el mercado отс, el cual sí genera riesgo de contraparte.

\subsection{Cuadrante II}

\subsubsection{Desarrollo del mercado}

En lo que respecta a normatividad, el mercado de derivados colombiano ha desarrollado nuevas políticas y normas buscando incentivar la negociación de los instrumentos financieros. Estas normas han facilitado y mejorado el desempeño del mercado, permitiendo así la creación de nuevos contratos que generan profundización y creadores de mercado para contratos específicos. La estrategia que se plantea consiste en implementar un marco normativo basado en los principios que la Comisión Nacional Bancaria y de Valores (2012) propuso para el mercado mexicano, los cuales coadyuvaron a la estabilidad financiera y generaron impactos positivos en el dinamismo y transparencia del mercado de derivados en México; estos principios son:

- Riesgo sistémico: se busca reducir por medio de la normatividad implementada el impacto que genera en el sistema financiero el incumplimiento de algún participante del mercado. Se exige la divulgación oportuna de la información del mercado.

- Mitigación de riesgos: busca reducir el riesgo de crédito de las operaciones liquidadas en cámaras centrales de compensación; asimismo, una regulación más fuerte para las negociaciones que no se liquiden en estas entidades.

- Transparencia: exige transparencia en la información posnegociación, con el fin de disminuir las controversias por términos y condiciones de las operaciones y mejorar la formación de precios en los sistemas de negociación.

- Eficiencia operativa: normatividad enfocada a facilitar el procesamiento automático de las operaciones y generar sistemas multilaterales y electrónicos de negociación.

- Mejores prácticas del mercado: busca que los participantes puedan acceder al mercado sin discriminaciones, evitando el uso de información privilegiada. 
Logros y retos de la implementación de derivados financieros entandarizados en colombia: un análisis comparativo con méxico, chile y perú, 2008-2012

\subsubsection{Desarrollo del producto}

Colombia debería aprovechar el escenario de crecimiento en el que se encuentra su mercado de derivados estandarizado a fin de estimular a los inversionistas por medio de la creación de contratos diferentes a los futuros, como son las opciones y los swaps financieros. Teniendo en cuenta la experiencia de México y la liquidez del mercado de capitales colombiano, se recomienda que el país avance hacia la implementación de opciones financieras, ya que este instrumento constituye una valiosa herramienta de cobertura para mejorar el rendimiento de portafolio de los inversionistas, al permitir tomar la decisión de llevar a cabo o no la liquidación del contrato.

\subsubsection{Integración horizontal}

La búsqueda de integración con los principales mercados de derivados estandarizados de la región, permitirá el fortalecimiento del mercado nacional. Actualmente Colombia realiza esfuerzos por concretar interconexiones con importantes mercados como es el caso de Brasil, donde se busca la exploración y viabilidad de conectar las plataformas de negociación de sus mercados de renta variable, renta fija y derivados. Adicionalmente, es importante resaltar que además de México, países como Brasil y Panamá muestran gran interés por realizar integraciones con países de la región, lo que generaría mayores oportunidades de fortalecer el mercado de derivados por medio de alianzas estratégicas en el largo plazo.

Así, se ha identificado la necesidad de realizar integraciones con otros mercados para llegar a ser un jugador reconocido a nivel internacional, y por tanto llamar la atención de grandes conglomerados bursátiles. De manera individual, Colombia tan solo representa el 6.4\% del mercado latinoamericano (Ortíz , 2010). Igualmente, se evidencia que las exigencias de los inversionistas son cada vez mayores en cuanto a alternativas de inversión y menores costos de transacción, lo que explica en gran medida las integraciones que se vienen dando en el mercado de valores a nivel mundial.

\subsection{Cuadrante III}

\subsubsection{Acceso al mercado de derivados}

Pese a que Colombia -comparado con Chile, Perú y México- tiene una gran desventaja al contar con un impuesto de $14 \%$ sobre ganancias, debido a que Chile y México eximen del cobro de impuestos a los inversionistas extranjeros y Perú cobra tan solo el 5\%, el mercado de capitales colombiano se caracteriza por su liquidez y dinamismo, lo que genera que los inversionistas se vean atraídos en mayor medida por estos aspectos que por un cobro tributario.

De otro lado, para algunos expertos, Colombia debería reducir e incluso eliminar el impuesto sobre ganancia a la inversión extranjera para mejorar la competitividad frente a los demás mercado analizados; sin embargo, otros argumentan que el exceso de inversión extranjera genera procesos de revaluación de la moneda local afectando algunos sectores de la economía, especialmente a aquellos que realizan operación de exportación (González, 2013).

Por consiguiente, la propuesta que se plantea en cuanto al tema de impuestos, es otorgar beneficios tributarios a las pymes colombianas que deseen ingresar en el mercado de derivados financieros estandarizado del país, ya que los volúmenes de negociación que se han registrado en el mercado desde su creación y que han sido impulsados por especuladores y por inversionistas extranjeros, ratifican que para ellos existen otros factores que pesan más que la carga impositiva que genera Colombia para la inversión extranjera. Según Lipsky (2010), en su artículo "Managing Financial Innovation in Emerging Markets", "Un acceso limitado al mercado de derivados exige a empresas a soportar costos que podrían evitar; esto puede afectar su capacidad de expansión".

\subsubsection{Estrategia a nivel de negocios}

Aquí se propone el desarrollo de un plan estratégico del mercado de derivados colombiano tomando como referencia el plan realizado por MexDer y Asigna (MexDer; 
2010, p. 9). Los objetivos estratégicos del plan giran en torno al posicionamiento del mercado, el ofrecimiento de soluciones automatizadas para la operación, liquidación y monitoreo del mercado, así como la participación activa de miembros y clientes finales tanto locales como extranjeros. Con el desarrollo del plan se busca incrementar el número de participantes, adecuar, integrar y reforzar la estructura operativa, consolidar los productos actuales, desarrollar y adaptar productos y reglas acordes con las necesidades del mercado

\section{Conclusiones}

En la investigación se han analizado los logros y los retos del mercado de derivados financieros estandarizado, desde su creación en 2008 hasta 2012, encontrando que Colombia tiene un gran potencial para desarrollar este mercado, ya que presenta crecimientos de más de $1000 \%$ en los volúmenes de negociación. Colombia se convirtió en el tercer país en lanzar el mercado de derivados en bolsa después de Brasil y México; pese a no tener la misma experiencia que estas bolsas, logró crecimientos superiores a los que presentó México, mercado que en promedio decreció un 11\% para los años estudiados.

Respecto al marco normativo en este mercado, uno de los principales logros fue la adaptación de la normatividad a principios de 2011, lo cual permitió la inserción de nuevos tipos de contratos como futuros sobre la acción de Ecopetrol y Pacific Rubiales y futuros sobre el índice accionario COLCAP.

Ahora bien, pese a los importantes avances en la diversificación de contratos de futuros, uno de los principales retos a los que se enfrenta el mercado de derivados estandarizado colombiano es el fortalecimiento e implementación de opciones y swaps, lo que permitirá ampliar la oferta de instrumentos negociados en bolsa atrayendo mayor inversión y generando la posibilidad que en el mediano plazo se llegue a tener volúmenes de negociación similares a los que presenta el mercado mexicano, los cuales en promedio son 50 millones de contratos negociados al año.
La investigación permitió constatar el bajo desarrollo que presenta Chile y Perú en cuanto al mercado de derivados estandarizados, aunque se resalta los múltiples esfuerzos que han realizado en lo que respecta a alianzas estratégicas buscando el fortalecimiento y profundidad del mercado.

Se afirma así la hipótesis planteada para el desarrollo de la investigación identificando que el mercado de derivados colombiano presenta un gran potencial para el desarrollo de los mercados financieros del país, no sólo por las cifras de crecimiento que ha presentado desde su creación, sino por el interés que ofrecen grandes plataformas de negociación como México y Brasil en la interconexión de los mercados tanto de renta variable, renta fija, derivados y la atracción que presenta Colombia frente a los demás países de la región para los inversionistas extranjeros.

\section{Referencias}

Banco de la República de Colombia. (06 de octubre de 2003-2012). Banco de la República de Colombia. Obtenido de http://www.banrep.gov.co/informeseconomicos/ine_inf_opderi.htm

Bernedo \& Azareño (16 de mayo de 2003). Banco Central de Reserva Del Perú. Obtenido de http://www. bcrp.gob.pe/docs/Publicaciones/Revista-Estudios-Economicos/09/Estudios-Economicos-9-5. pdf

Budnevich y Asociados (28 de agosto de 2009). Superintendencia de Bancos e Instituciones Financieras (SBIF). Obtenido de http://www.sbif.cl/sbifweb/internet/archivos/publicacion_9143.pdf

Comisión Nacional Bancaria y de Valores (noviembre de 2012). CNBV. Obtenido de http:/www. iimv.org/actividades2/Antigua2012-2/martes $13 /$ LuisLeyvaE1\%20Mercado\%20de\%20 Mercado\%20de\%20Derivados\%20Mexico\%20 y\%20Reformas \%20al\%20OTC_LLM_\%20 12 NOV2012\%20IIMV\%20LA \% 20 ANTIGUA\%20GUAT.pdf 
Logros y retos de la implementación de derivados financieros entandarizados en colombia: un análisis comparativo con méxico, chile y perú, 2008-2012

Comisión Nacional Bancaria y de Valores (2012). Normatividad. Obtenido de http://www.cnbv.gob.mx/ Paginas/Normatividad.aspx

Córdoba (01 de septiembre de 2008). "Colombia tercer país en lanzar mercado de derivados”. En: El Espectador, p. 1 .

Deceval y Anif. (2009). "Desarrollo del mercado de derivados en colombia. En: Enfoque mercado de capitales, 1-3.

Díaz, T. \& Hernández, T. (2003). Futuros y opciones financieras: Una introducción. México: Limusa.

Fred, R. (2003). "Conceptos de Administración estratégica: Cap. 5”. En: Estrategias en acción ( $9^{\circ}$ ed.). México: Pearson

Gómez, J. (2010). Dinero, banca y mercados financieros. Bogotá: Alfaomega.

González, M. (30 de enero de 2013). “"Impuesto golondrina', ¿Bueno o malo para la economía?” En: $L a$ Prensa Libre.

Hull, J. (2002). Introduccion a los mercados de futuros y opciones completo. Madrid: Prentice Hall.

Jiménez, E. (13 de mayo de 2012). Asopymes. Obtenido de http://asopymescolombia.org/blog/ la-importancia-de-las-pymes/
Lipsky, J. (2010). "Managing Financial Innovation in Emerging Markets". En: MF Remarks at RBI First International.

MexDer(05 de marzo de 2010). Mexder una Introducción. . Obtenido de http://www.mexder.com.mx/wb3/ wb/MEX/MEX_Repositorio/_vtp/MEX/1ef6_publicaciones/_rid/21/_mto/3/UnaIntroduccionESP. pdf

Ochoa \& Benjerodt (01 de mayo de 1996). Banco Central de Chile . Obtenido de http:/www.bcentral.cl/estudios/estudios-economicos/pdf/serieestudios 40 . pdf

Saavedra y Utrilla. (21 de mayo de 2010). Universidad Autonoma de Mexico . Obtenido de http://www. paginaspersonales.unam.mx/files/210/Derivados. pdf

Salazar, G. (2008). "Mercado de derivados". En: Carta Financiera, 22-28.

Universidad Nacional de Manizalez (2012). "Matriz de la Gran Estrategia". obtenido de; http:/www.virtual. unal.edu.co/cursos/sedes/manizales/4010014/ Contenidos/Capitulo5/Pages/5.9/592Segunda etapa.htm 